\title{
New Challenges for Theoretical Computer Science
}

\section{(General Introduction to the Panel)}

\author{
Jozef Gruska \\ Faculty of Informatics, Masaryk University, \\ Botanická 68a, 60200 Brno, Czech Republic \\ gruska@www.fi.muni.cz
}

\begin{abstract}
This is a general introduction to the panel New Challenges for Theoretical Computer Science with panelists G. Ausiello, J. Gruska, U. Montanari, T. Nishizeki, Y. Toyama and J. Wiedermann. The aim of the panel is to point out and analyse new challenges theoretical computer science has to deal with in order to develop properly as fundamental science and in order to respond well to needs coming from the outside world, especially to those informatization of society and global networking bring.
\end{abstract}

Vision Theoretical computer science still keeps being developed too much according to the same principles as pure mathematics - following mainly the inherent needs to develop more powerful concepts and proof methods, to generalize results, to simplify proofs, and to solve important/interesting or long standing problems. Most of the pure mathematicians hardly look to the outside world for its needs and motivations.

Theoretical computer science is believed here to have some needs to follow the same research paradigms as mathematics does, but to have ultimately also much broader goals: To study fundamental laws and limitations of the information processing world and also to contribute to the development of the new, the third, main methodology 11 of mankind in general and of science in particular. Moreover, theoretical computer science has a need and obligation to make its contribution to solving new big and difficult problems informatization and global communication bring. Theoretical computer science therefore needs more often and more carefully to look out of its theoretical vacuum to see whether it is concentrating correctly and enough on its main global aims, to see where the problems are it can and should help to solve and how really powerful are tools it develops.

\footnotetext{
* Support of the GAČR grant 201/98/0369 and of the grant CEZ:J07/98:143300001 is to be acknowledged.

${ }^{1}$ This refers to the understanding that so far there were two main methodologies science had: the theoretical one (much represented and supported by mathematics) and the experimental one. Computer Science is believed to bring a third methodology that at the same time reinforces (substantially) old methodologies and helps to bridge them.
} 
Challenges At the break of the millennia theoretical computer science is faced with several new, scientifically fundamental and practically very important and hard challenges.

- Due to several developments one can witness a liberalization of theoretical computer science from serving mainly computation and communication technology. Theoretical computer science becomes more and more clearly fundamental science that has to study the laws and limitations of the information processing world in all its forms and it has to concentrate on developing corresponding foundations, paradigms, concepts, models and tools.

- An understanding of the limitations of current computing and communication technologies and methods has turned attention to a more systematic study of the ways Nature can perform information processing. The goals are two sided: To develop new, more Nature based, information processing tools and also to get a better understanding of Nature. Quantum computing, molecular computing and brain computing are among areas that keep bringing new challenges fascinating in both directions.

- Radically new questions concerning computing and its power starts to be seriously asked and radically new theories and computational models need and start to be on the agenda. They range from theories and models connected with new applications and new computational modes to theories and computational models that try to understand brain, to be more powerful than "Turing models", to capture the essence of learning and understanding and even to capture the essence of evolution and genesis of Universe. And not only that. Similarly as in physics the question "Is there a (computational) model of everything" is being raised.

- Since society is increasingly dependent, see [2], on very large software systems and on global communications, reliability of very big software systems and security of communications are becoming another very hard and very important problems theoretical computer science is asked and obliged to help to deal with - by developing corresponding insights, concepts, theories and methods.

- The existence of global networks and their size and complexity require to develop new theoretical tools to handle new issues created by them. For example, the task is to develop abstract semantics framework to deal with such problems of highly distributed networks as those created by needs to manage cooperation, control and synchronization as well as verification of systems with high mobility of codes (created in different programming languages) and computations.

- Various limitations concerning information processing have been discovered. Some of them are very unpleasant. For example enormous number of practically important problems are NP-complete. An important task is to find ways how to compute, in some reasonable sense, what cannot be computed in some very strict sense and worst case.

- A variety of new challenges for theoretical computer science come also with fast growing needs to solve (efficiently) problems with enormously large data. 
New dimensions to this problem are due to the facts that these data may be produced in a very distributed way and can keep being dynamically changed. In addition, they need to be solved in an on-line way. This in turn brings to a new dimension problems of efficient distributed computing and of efficient use of hierarchies of (secondary) memories. Searching for textual, visual and sound data in wide area networks, with millions of nodes and allto-all connections, as well as broadcasting of such data in global networks are examples of such problems.

Some of these challenges are discussed in more details in position statements of panelists.

Teaching of theoretical computer science seems to be in a crises. Students keep challenging relevance of some subjects and ways theory is presented. Theoretical computer science seems to have needs to learn from other mature sciences, as physics, chemistry and biology to present its concepts, methods and results in much more synthetizing form and to concentrate mainly on outcomes with clear importance (see [1], for an attempt to do that). New challenges theoretical computer science is to deal with require also that its fundamental and powerful of concepts, methods and results are presented to students in a much more mature and impressive way.

Epilogue Theoretical computer science research should get out of its "concentration on $\lg ^{*} n$ improvements" and its education should be such that students do not remember it mainly as a "pumping-lemmas stuff". Big new challenges of theoretical computer science and enormous potentials it has require that.

\section{References}

1. Jozef Gruska. Foundations of computing. Thomson International Computer Press, 1997.

2. President's Information Technology Advisory Committee. Information. Informaation technology research: Investing in our future. Technical report, Report to the President, National Coordination Office for Computing, Information and Communication, 1999. 